\title{
Understanding EDXS Analysis of Nanostructures in TEM
}

\author{
Huafang $\mathrm{Li}^{1}$, Parag Banerjee ${ }^{1}$ and Kathy Flores ${ }^{1}$ \\ ${ }^{1 .}$ Institute of Materials Science and Engineering, Washington University in St. Louis, St. Louis, \\ Missouri, USA
}

EDXS elemental identification and quantification is a routine practice in materials characterization. In TEM, elemental quantification through EDXS is limited by the unknown absorption process in the material. Generally Cliff-Lorimer ratio method and absorption correction has been used for quantification $[1,2]$, based on assumptions of column electron beam and parallel sample surface. However, for nanostructures such as nanoparticles and nanorods, parallel surface assumption is invalid, the X-ray path to the detector depends on the geometry of the nanostructures and location of the detector. In this paper, we introduce a new model for elemental quantification. As examination sample to verify the model, $\mathrm{ZnO}$ single crystalline nanorods are used. The $\mathrm{ZnO}$ nanorods are suspended on lacy carbon coated copper TEM grid. Scanning transmission electron microscopy (JEOL JEL-2100F TEM) and Bruker Quantax microanalysis system (XFlash Detector) are used in this study.

The intensity (counts) of X-ray $(I)$ that read from detector depends on the X-ray generated $\left(I_{G}\right)$ from electron beam bombardment, absorption ratio $\left(R_{A}\right)$ of materials during the path to detector and detector collection factor $\left(R_{D}\right)$. The intensity can thus be expressed as the following:

$I=I_{G}\left(1-R_{A}\right) R_{D}$

The amount of $\mathrm{x}$-ray generated depends on electron source, material property (how easy to be ionized to emit the specific x-ray upon the bombardment) and interaction volume. The absorption ratio depends on the $\mathrm{x}$-ray absorption coefficient and the $\mathrm{x}$-ray path to the detector. The ionization ratio of material to emit the specific $\mathrm{x}$-ray can be defined as $k$. The absorption ratio $R_{A}$ is a function of x-ray path to the detector $t^{\prime}$.

$R_{A}=A t^{\prime}+B t^{\prime 2}+C t^{\prime 3}+\cdots \quad$ (A, B, C are the absorption constant of a material)

To correlate $t^{\prime}$ to sample thickness $t$, we define the geometry correction $\Delta G=t^{\prime} / t$. As the thickness of specimen is small, higher order absorption can be ignored. For electron probe with diameter $\mathrm{D}$ goes through the specimen with thickness $t$, Equation (1-1) can be modified as:

$I=k\left(\frac{1}{4} D^{2} t\right) \cdot[1-A(t \cdot \Delta G)] \cdot R_{D}$

When the electron probe diameter and detector factor is fixed, for a specific material, the counts $I$ read from the EDXS detector is varied by the specimen geometrical shape. Now if we use the ratio method, for x-ray with energy $E_{A}$ and $E_{B}$,

$\frac{I_{E_{A}}}{I_{E_{B}}}=\frac{k_{E_{A}}\left(\frac{1}{4} D^{2} t\right) \cdot\left[1-A_{E_{A}}(t \cdot \Delta G)\right] \cdot R_{D\left(E_{A}\right)}}{k_{E_{B}}\left(\frac{1}{4} D^{2} t\right) \cdot\left[1-A_{E_{B}}(t \cdot \Delta G)\right] \cdot R_{D\left(E_{B}\right)}}=\frac{k_{E_{A}}\left[1-A_{E_{A}}(t \cdot \Delta G)\right] \cdot R_{D\left(E_{A}\right)}}{k_{E_{B}}\left[1-A_{E_{B}}(t \cdot \Delta G)\right] \cdot R_{D\left(E_{B}\right)}}$

Considering $E_{A}$ and $E_{B}$ are the characteristic x-ray of element $\mathrm{A}$ and element $\mathrm{B}$, the equation can be related to the composition $C_{A}$ and $C_{B}$,

$\frac{I_{A}}{I_{B}}=K_{A, B} \cdot \frac{C_{A}}{C_{B}} \cdot \frac{\left[1-A_{A}(t \cdot \Delta G)\right]}{\left[1-A_{B}(t \cdot \Delta G)\right]}$

$K_{A, B}$ is the sensitivity factor. $(t \cdot \Delta G)$ is the geometric factor $(G)$ of the specimen. Thus, 
$\frac{I_{A}}{I_{B}}=K_{A, B} \cdot \frac{C_{A}}{C_{B}} \cdot \frac{1-A_{A} \cdot G}{1-A_{B} \cdot G}$

Equation (1-4) indicates that the x-ray counts ratio is varied by the geometry of the specimen; it is valid even when column assumption of electron beam is inappropriate and the $\mathrm{x}$-ray path to the detector is not same from the places that X-ray generated. It is therefore suitable for both material with small dimensions and bulk material.

Figure 1 (a) shows a typical line scan EDXS measurement of $\mathrm{ZnO}$ nanorod. As there is no compositional difference consideration, the different change in the counts of $\mathrm{Zn} \mathrm{K} \alpha$ and $\mathrm{Zn} \mathrm{L}_{\alpha}$ lines is due to the absorption. The counts ratio of $\mathrm{Zn} \mathrm{K}_{\alpha}$ and $\mathrm{Zn} \mathrm{L}_{\alpha}$ can be used to determine the geometric factor based on equation (1-4). Figure 1 (b) shows the $\mathrm{Zn}: \mathrm{O}$ compositional ratios calculated from Cliff-Lorimer method and absorption correction based on equation (1-4). The declination of $\mathrm{Zn}: \mathrm{O}$ ratio along the scanning distance from Cliff-Lorimer method can be corrected using our model. The absorption constants $A$ used are $A_{\mathrm{Zn} \mathrm{K}}: A_{\mathrm{Zn} \mathrm{L}}: A_{\mathrm{O}}=5400: 287000: 600000$, calculated from reference [3]. The ratio relationship of absorption constant $A$ can be determined experimentally, or calculated from mass absorption coefficient. The sensitivity factors are $K_{\mathrm{Zn}}$ $\mathrm{K}: K_{\mathrm{Zn}}: K_{\mathrm{O}}=5.557: 1.561: 1.695$ for Cliff-Lorimer method and $K_{\mathrm{Zn} \mathrm{K}}: K_{\mathrm{Zn} \mathrm{L}}: K_{\mathrm{O} \text { K}}=5.6: 4.6: 2.9$ for absorption correction.

This paper introduces a new model about EDXS elemental quantification. Different from other absorption correction methods, this model need not estimate $\rho t$ of the material. The model can also be used to examine the sensitivity factor $K_{\mathrm{A}, \mathrm{B}}$ and the absorption constant ratio of material.

\section{References:}

[1] DB Williams and CB Carter, Transmission Electron Microscopy, 1996 Plenum Press, New York. Section IV, Spectrometry.

[2]Conference Proceedings (1981), “Quantitative Microanalysis with High Spatial Resolution”, (The Metals Society, London).

[3] EB Saloman and JH Hubbell, Atomic Data and Nuclear Data Tables 38 (1988), p.1.
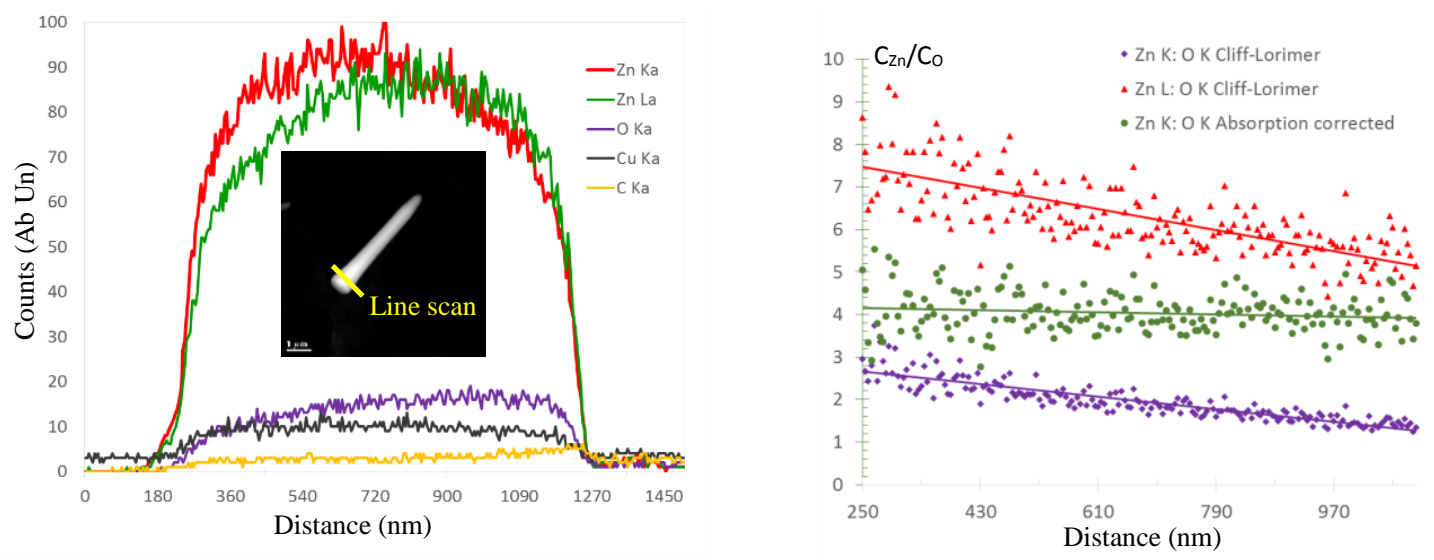

Figure 1. (a) EDXS line scan of a $\mathrm{ZnO}$ single crystalline nanorod, (b) Compositional ratio calculated using Cliff-Lorimer method, and through absorption correction using the model proposed in this study. 